\title{
Asimetría Fluctuante: Una herramienta morfo-funcional para Medir Estabilidad del Desarrollo
}

\author{
Fluctuating Asymmetry: A Morpho-functional Tool to Measure Development Stability
}

${ }^{*, * * * H u g o ~ A . ~ B e n i ́ t e z ~ \& ~ * L u i s ~ E . ~ P a r r a ~}$

BENITEZ, H. A. \& PARRA, L. E. Asimetría fluctuante: una herramienta morfo-funcional para medir estabilidad del desarrollo. Int. J. Morphol., 29(4):1459-1469, 2011.

RESUMEN: La capacidad de un organismo para producir un fenotipo ideal, pese a las perturbaciones encontradas durante su desarrollo, es el mecanismo causal de la estabilidad del desarrollo (ED). Esta capacidad es utilizada para evaluar variedad de tipos de estrés y la capacidad genotípica de corregirlos. La herramienta de medición más utilizada para estimar la ED es la asimetría fluctuante (AF), siendo esta una medida de las pequeñas desviaciones al azar que ocurren entre el lado izquierdo y derecho de rasgos bilateralmente simétricos. El estudio de AF, por su simplicidad de manejo y rapidez en la obtención de resultados, se ha convertido en un foco de interés para la biología, y durante los últimos años el aumento en artículos publicados usando AF para evaluar perturbaciones en la ED ha sido progresivo, pero aun así existen muchas interrogantes de cuales son las causas subyacentes que generan la AF. La presente revisión recopila información sobre el avance en los estudios del uso de la AF, su metodología, mecanismos, ventajas y controversias en el tiempo, y propone que es estrictamente necesario generar una mejor comprensión de esta herramienta, producto de eso, la utilización de modelos más exactos de estudio que signifiquen (o justifiquen) un enfoque unificado en el análisis de los patrones en AF y ED.

PALABRAS CLAVE: Inestabilidad del desarrollo; Selección Sexual; Genotipo; Fenotipo.

\section{INTRODUCCIÓN}

La estabilidad del desarrollo (ED) se define como la capacidad de un organismo para producir un fenotipo predeterminado por un diseño corporal adaptativo bajo un conjunto de condiciones genéticas y ambientales específicas (Waddington, 1942). Se refiere, por tanto a las capacidades intrínsecas de un individuo para resistir accidentes y perturbaciones durante su crecimiento y desarrollo (Clarke, 1998).

La capacidad de un organismo para producir un fenotipo ideal, pese a las perturbaciones encontradas durante el desarrollo, reflejan el mecanismo causal de la ED. Esta capacidad es utilizada para evaluar variedad de tipos de estrés y la capacidad genotípica de corregirlos (Auffray et al., 1999). A menudo se denomina inestabilidad del desarrollo (ID) o ruido del desarrollo (RD) a la tendencia de un sistema del desarrollo para producir cambios morfológicos como respuestas a perturbaciones aleatorias (Klingenberg, 2003; Nijhout \& Davidowitz, 2003; Van Dongen, 2006).

La herramienta de medición más utilizada para estimar la ED es la Asimetría Fluctuante (AF) (Palmer \&
Strobeck, 1986; Palmer, 1994; Clarke, 1998; Pither \& Taylor, 2000). La AF es una medida de las pequeñas desviaciones al azar que ocurren entre el lado izquierdo y derecho de rasgos bilateralmente simétricos (Van Valen, 1962). La principal idea en el uso de la AF como medida de ID, es que el lado izquierdo y derecho (I - D) de un organismo puedan ser vistas como replicas independientes de un mismo desarrollo, donde los lados corporales de un individuo compartirían un mismo genotipo en un ambiente homogéneo (Klingenberg, 2003). En otras palabras, los factores determinantes del desarrollo del lado I y D serian idénticos, por lo que en condiciones ideales se esperaría para éstos una simetría perfecta (Auffray et al.; Klingenberg, 2003; Van Dongen, 2006). Sin embargo, en el proceso natural del desarrollo de un organismo, casi siempre existen pequeñas perturbaciones de tipo celular (ruido del desarrollo) (Polak, 2003). Como estas perturbaciones se producen en una pequeña parte del organismo, se espera que los efectos se acumulen de forma independiente en el lado izquierdo y derecho (Klingenberg).

\footnotetext{
* Departamento de Zoología, Facultad de Ciencias Naturales y Oceanográficas, Universidad de Concepción. Concepción, Chile.

** Instituto de Alta Investigación, Universidad de Tarapacá, Arica, Chile.
} 
Esto quiere decir que la asimetría visible y cuantificable de los lados (I-D) de las estructuras morfológicas sería por tanto la expresión de las perturbaciones que se han acumulado durante el desarrollo. El aumento de estas perturbaciones afecta la expresión del organismo sometido a niveles de estrés durante el desarrollo o su capacidad limitada de corregirlas. De otro modo se asume que la simetría es óptima, y que cualquier desviación de esta estaría constituyendo un grado de inestabilidad (Palmer \& Strobeck, 1986).

El estudio de AF, por su simplicidad de manejo y rapidez en la obtención de resultados, se ha convertido en un foco de interés para la biología. La mayoría de los estudios en ecología, biología evolutiva y genética, entre otros, utilizan AF como medida directa de la estabilidad del desarrollo, ruido del desarrollo o inestabilidad del desarrollo (Klingenberg) (Fig. 1).

Durante los últimos años, y en un afán de descubrir algunas de estas interrogantes, el incremento en artículos publicados usando AF para evaluar perturbaciones en ED ha sido progresivo. Los registros de ISI Web of Knowledge a la fecha señalan 2088 artículos publicados, con un aumento exponencial en la última década que llega a más de 130 artículos anuales (Fig. 2).

El presente artículo recopila información histórica del avance de AF como herramienta para medir estabilidad durante el desarrollo, sus mecanismos, ventajas y controversias.

\section{Metodología: Análisis de AF: validación de los datos}

a) Tamaño de muestra en AF. Las pruebas estadísticas para evaluar diferencias en AF son las pruebas que calculan las diferencias en las variaciones entre muestras. Incluso usando un test $\mathrm{F}$ para probar si existen diferencias en $\mathrm{AF}$ entre dos muestras, la capacidad para detectar -por ejemplo una diferencia en la variación- depende en gran medida del tamaño de la muestra. Un tamaño muestral de 10 ejemplares sólo manifiesta una diferencia significativa del 25\% (a = 0,05 ), un tamaño de muestra de 25 ejemplares revelaría diferencias significativas en el 50\% de las veces, y un tamaño de muestra de 40 ejemplares indicaría diferencias significativas del $75 \%$ de las veces.

Como en cualquier análisis estadístico, el tamaño de la muestra recomendado depende de la magnitud de las diferencias en la "señal" (en este caso AF) entre muestras. Como regla empírica, $\mathrm{n}=30$ se considera un tamaño mínimo de la muestra en los estudios de AF. Cuando existe inquietud en relación de las posibles desviaciones de la normalidad, un "n" de 40 ó 50 sería más deseable. El tamaño de la muestra y el error influyen mucho en la capacidad de detectar desviaciones de la normalidad (Palmer, 1994).

b) Elección de caracteres. Generalmente la mayoría de los trabajos publicados se centran en evaluar características de fácil medición, y esto se debe a que una medición expedita reduce los niveles de error y por lo tanto facilita el cálculo de asimetrías más precisas. La pregunta es: ¿Son estos caracteres realmente significativos o existen otras variables

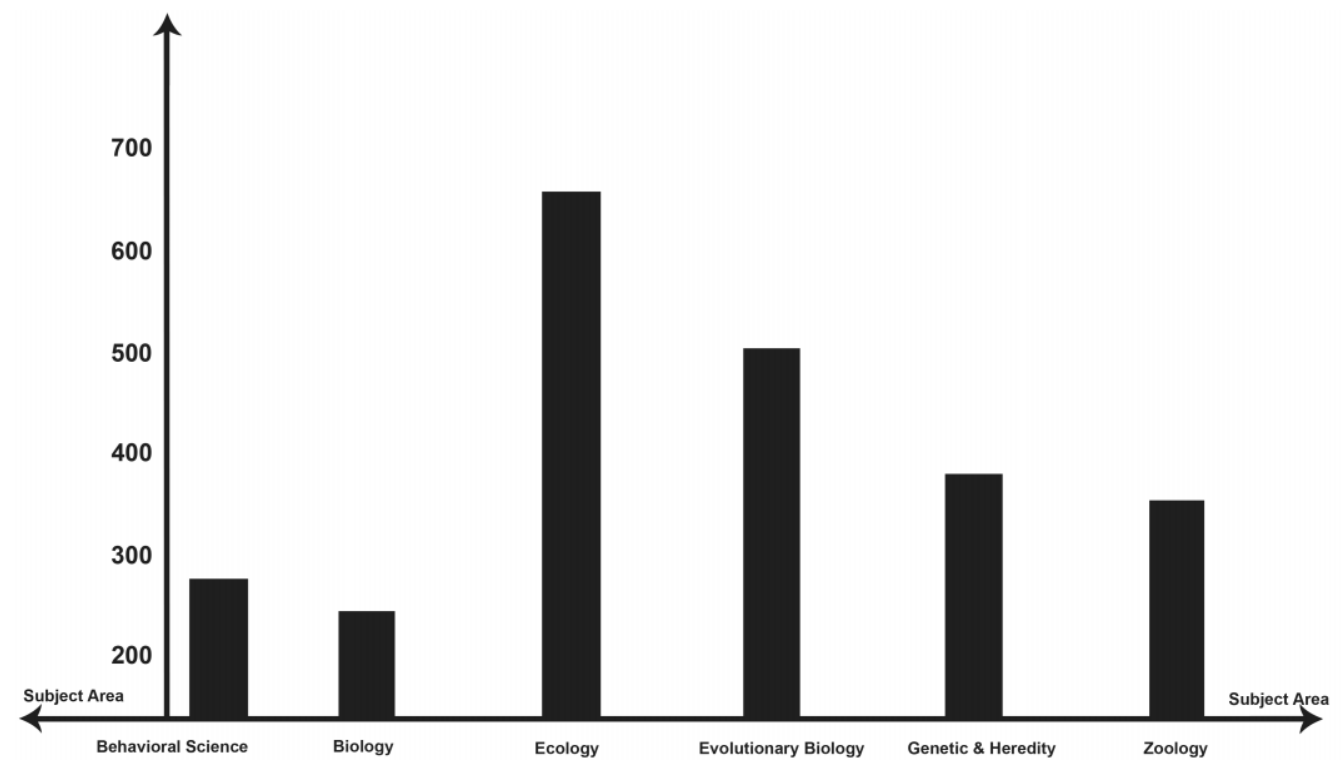

Fig. 1. Cantidad de artículos publicados a la fecha por área temática en AF 


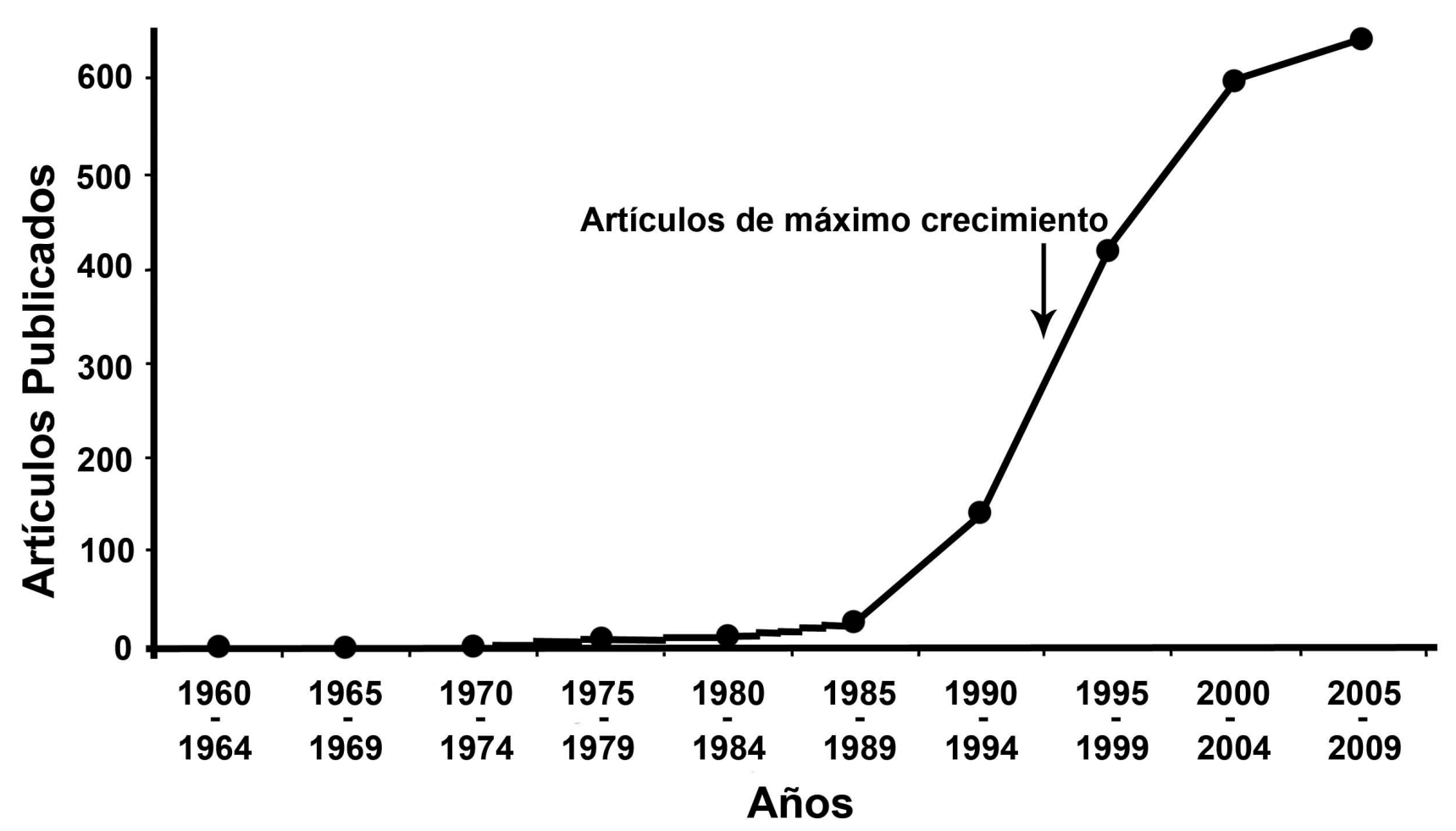

Fig. 2. Número de artículos en AF publicados a la fecha (registros de ISI Web of Knowledge)

de mediciones más complejas pero de mayor interés? Estudiar el desarrollo de rasgos correlacionados, puede llegar a ser una de las formas más complejas de medir variables (eg. Klingenberg \& McIntyre, 1998; Mardia et al., 2000; Klingenberg et al., 2001). En estos ejemplos particulares, los autores analizan la variación de la forma global del ala del insecto y como su asimetría puede ser relevante en el comportamiento del vuelo. Para esto utilizaron herramientas de morfometría de landmarks (Procustes) y evaluaron la variación de la forma y simetría en diferentes planos, tanto en dos dimensiones como en tres dimensiones (Swaddle, 2003).

La evaluación de caracteres correlacionados realizaría una estimación más precisa al momento de medir la inestabilidad del desarrollo que un rasgo individual (Polak \& Starmer, 2001). Esto es muy significativo, ya que la técnica prueba la misma inestabilidad del desarrollo pero con más de dos puntos de datos. Combinar la medición de asimetría de rasgos múltiples estandarizado en un solo índice (ver más adelante) (Windig \& Nylin, 2000) podría ser un indicador total de asimetría corporal (Woods et al., 1999). Sin embargo, y debido a que AF es un tanto específica de un rasgo, no hay claridad sobre la medida que indicaría si los rasgos estas correlacionados y ligados al desarrollo. Aun cuando todas las medidas de asimetría estén relacionadas con la inestabilidad del desarrollo, los diferentes rasgos pueden tener diferentes capacidades variando la susceptibilidad de estos a factores de estrés ambiental (producto de la heterogeneidad de las diferentes vías bioquímicas en el desarrollo) y los distintos patrones ontogenéticos o tiempos de desarrollo de la asimetría (Swaddle, 2003). Por lo tanto, la interpretación de índices multirasgos de asimetría (cuando estos rasgos no están relacionados con el desarrollo) podría ser más problemático que la interpretación de los rasgos individuales.

c) Índices Convencionales de AF. Para calcular y representar numéricamente los valores de AF a nivel individual o poblacional, se han descritos un alto número de índices (Palmer \& Strobeck 1986; 2003). Sin embargo, solamente algunas variables de los índices son realmente fundamentales. Palmer (1994) indicó que la asimetría de un carácter determinado en un individuo se mide como el valor absoluto de la diferencia entre lados derecho e izquierdo (|Di- Ii $\mid)$, como la diferencia asignada $(\mathrm{Dj}$ - $\mathrm{Ij}$ ), o como la relación entre las mediciones de cada lado de un individuo (D/Ii) (Palmer \& Strobeck, 1986). Cada una de estas mediciones se han utilizado como base de los índices para analizar las diferencias entre la asimetría de las muestras, en las que la principal diferencia es la presencia o ausencia, o como se maneja la variación en el tamaño de un carácter. Otro índice que evalúa múltiples rasgos se define como: 1 - r, donde " $r$ " es la correlación entre los lados izquierdo y derecho. Windig \& Nylin argumentan que el índice 1 - r es útil en las comparaciones de caracteres, porque: a) es independiente de la 
escala; b) puede corregir el error de medición; y c) incluye un índice normalizado por la varianza del rasgo.

Los dos índices más frecuentemente usados en literatura son FA1 y FA4. Una modificación común para estos índices es corregir por dependencia de tamaño dividiendo por el tamaño del carácter principal, ya sea a nivel de individuos (FA2, FA6, FA8) o a nivel de muestras (FA3, FA7; ver sección 10 Palmer, 1994). Otro índice (FA10) se obtiene de los resultados de un procedimiento ANDEVA que prueba simultáneamente los errores de medición y diferencias de tamaño entre individuos.

d) Error de Medición en AF. Dentro de los factores que predicen la estabilidad del desarrollo, la desviación de la simetría mantiene una alta incertidumbre debido a dos fuentes de error: a) el error de medición (EM); y b) el error de muestreo. Generalmente, el esfuerzo para calcular EM es bajo y, a diferencia de los análisis convencionales, en donde EM sólo reduce la señal en relación con el ruido, EM plantea graves problemas para los análisis de $\mathrm{AF}$ (Greene, 1984; Palmer \& Strobeck, 2003; Swaddle et al., 1994, Fields et al., 1995; Merilä \& Björklund, 1995; Fumi, 2008; MuñozMuñoz \& Perpiñan, 2010). Las pequeñas desviaciones de los planos de simetría suelen ser semejantes en magnitud a los EM, éste contribuye en un alto porcentaje de la variación en un carácter, y reduce la correlación observada entre AF e ID subyacente (Palmer, 1996; Van Dongen \& Lens, 2000). Para corroborar que las diferencias entre los lados D - I en individuos diferentes no es un efecto del EM, la relación entre AF y EM se debe evaluar. Además para asegurar que las diferencias entre D-I reflejen realmente las diferen- cias entre individuos, y que ésta no sólo se debe a un error de muestreo, se necesita la estimación de la repetitividad hipotética (Van Dongen, 1998). En caracteres métricos el error de medición suele ser inevitable.

Para calcular el error de medición, Palmer \& Strobeck (1986) describen una ANOVA de dos vías (lado x individuo, ver Tabla IIIa en Palmer, 1994), este cálculo debe realizarse como parte de cualquier estudio AF. Si el efecto de la interacción no es significativa (Tabla I), las pruebas de las diferencias AF entre muestras no estarían justificadas. Es importante considerar que esta prueba de ANOVA es utilizable para todas las variaciones entre lados relacionados con EM, incluyendo AF y otros tipos de asimetrías. En otras palabras, esta prueba evalúa si la diferencia entre los lados varía más entre individuos de lo que sería esperable, dado el tamaño del error de medición.

Asimetría fluctuante y Stress. La AF ha sido ampliamente utilizada para comparar los niveles de ID en poblaciones sometidas a una serie de factores de estrés ambiental, tales como: temperatura, nutrición, radiación, productos químicos, la densidad poblacional, efectos sonoros, parasitismo, condiciones de luz, riesgo de depredación y la estructura del hábitat (Hoffmann \& Woods, 2003; Møller \& Swaddle, 1997). Las hipótesis propuestas para evaluar los diferentes niveles de estrés ambiental, indican que ID y los resultados esperables, se observarían en altos niveles de AF en poblaciones más expuestas, a diferencia de un "control", que para poblaciones poco expuestas (Pankakoski et al., 1992, Graham et al., 2000). La aseveración sobre si AF es un indicador general y sensible del estrés del desarrollo es una de

Tabla I. Componentes de varianza en un modelo ANOVA factorial de dos vías (Palmer, 1994)

\begin{tabular}{lcccc}
$\begin{array}{l}\text { Fuente de } \\
\text { variación }\end{array}$ & $\begin{array}{c}\text { Cuadrados } \\
\text { medios } \\
\text { abrev. }\end{array}$ & Df & $\begin{array}{c}\text { Cuadrados medios } \\
\text { esperados }\end{array}$ & $\begin{array}{c}\text { Cuadrados medios } \\
\text { usados para prueba. }\end{array}$ \\
\hline Lado & $M S_{s}$ & $(S-1)$ & $\sigma_{m}^{2}+M\left(\sigma_{i}^{2}+\left(\frac{J}{S-1}\right) \sum \alpha^{2}\right)$ & $\begin{array}{c}\text { Asimetría } \\
\text { Direccional } \\
\text { Tamaño /variación } \\
\text { de la forma* }\end{array}$ \\
Lado x Ind. & $M S_{j}$ & $(J-1)$ & $\sigma_{m}^{2}+M\left(\sigma_{i}^{2}+S \sigma_{j}^{2}\right)$ & $\begin{array}{c}\text { A simetría no } \\
\text { direccional ** }\end{array}$ \\
Error med. & $M S_{s j}$ & $(S-1)(J-$ & $\sigma_{m}^{2}+M \sigma_{i}^{2}$ &
\end{tabular}

$\mathrm{S}=$ número de lados $\mathrm{J}=$ número de genotipos (='individuos'), $\mathrm{M}=$ número de medidas repetidas. åa2= Componente de varianza fijo agregado debido a los lados. $\mathrm{s} 2 \mathrm{j}$ = Componente de varianza aleatorio agregado debido a "genotipos" (= individuos); éste refleja la variación en el tamaño del carácter total o la forma entre individuos). $\mathrm{s} 2 \mathrm{i}=$ Componente de varianza aleatorio agregado debido a la falta de asimetría direccional (refleja la variación en la diferencia entre lados de los individuos como AF u otro tipo de asimetría). s2m = Componente de varianza aleatoria debido al error de medición. * Indica la variación en el tamaño o la forma del carácter en general de los individuos, lo que puede ser útil para probar la eficacia de la escala del tamaño. ** Incluye todas las formas de asimetrías no direccionales, en este caso $\mathrm{AF}$ y otros tipos. 
las creencias más extendidas en la literatura sobre AF. AF sería un biomonitor ideal para identificar poblaciones sometidas a presiones ambientales o de otro tipo, debido a su aparente generalidad con respecto a las especies, caracteres y estrés (Parsons, 1992). Muchos autores indican que AF puede ser utilizada como un indicador confiable (Clarke, 1995a; 1995b), debido a su barata y fácil manera de medir en comparación con otros componentes tradicionales del fitness (Bjorksten et al., 2000).

Así como muchos estudios sugieren al estrés como principal factor que puede aumentar los niveles de AF, existen otros donde no hay ni siquiera efectos opuestos del estrés en AF (Leamy, 1999; Woods et al.). Un alto número de factores, tales como la incertidumbre en los grados de estrés y la elección de caracteres e índices de AF, representan potenciales causas de la inconsistencia entre el estrés y AF.

Los resultados de muchas pruebas de medición de estrés mediante AF muestran una alta inconsistencia, dado que no existe un patrón general para caracterizar al estrés. Una posible razón a esta aseveración es que la mayoría de los estudios son de correlación, y sus resultados generalmente son confusos por la acción agentes ambientales no controlados. La mayoría de estos estudios examinan diferencias de AF a nivel poblacional, sin tomar en cuenta que las poblaciones pueden diferir en muchos factores distintos del estrés ambiental específico. Un método más poderoso para este caso sería examinar los cambios en AF como respuesta a la manipulación experimental de estrés bajo condiciones controladas (Bjorksten et al.).

Asimetría fluctuante y Fitness. Si realmente AF es una medida de ID, sería normal imaginar que los individuos con niveles más altos de ID, podrían ser los menos aptos. Esto explicaría una relación negativa de AF con el fitness o condiciones individuales (Møller \& Thornhill, 1998; Thornhill et al., 1999; Leamy \& Klingenberg, 2005).

Según la hipótesis de selección sexual para AF, las desviaciones de la simetría bilateral perfecta revelarían diferencias en el fitness o en la calidad entre individuos (Møller \& Pomiamkowski 1993; Møller \& Swaddle; Tomkins \& Simmons, 2003, Benítez et al., 2008). Se sugiere que la presencia de $\mathrm{AF}$ en rasgos sexuales secundarios, indicaría la capacidad de los machos para luchar contra las condiciones ambientales durante el crecimiento y el desarrollo de su ornamentación (Møller, 1990). Algunos procesos que explican la evolución de los caracteres sexuales secundarios son: a) selección natural, explicada por competencia entre machos (competencia intrasexual); y b) selección sexual por parte de la hembra. Mediante el primer proceso las estructuras evolucionan como armas para utilizarlas durante la lucha entre machos por las hembras, que generalmente corresponde al sexo que escoge la pareja. Mediante el segundo proceso, las hembras pueden escoger a machos con determinados caracteres sexuales secundarios. Se entiende que la selección sexual se produce como resultado de una asociación no azarosa entre un carácter sexual secundario y un componente del éxito reproductivo (Møller, 1994). Los machos con determinados caracteres sexuales secundarios tendrán ventajas frente a otros machos, ya sea en la lucha por conseguir pareja o por ser seleccionados por una hembra, y por tanto dejarán más descendencia que heredará sus caracteres (Leamy \& Klingenberg).

En algunos casos, los individuos simétricos son seleccionados sobre sus competidores asimétricos en la elección de pareja o en la competencia intrasexual (Swaddle, 1999). Como la mayoría de estos estudios informan correlaciones entre parámetros de selección de pareja y asimetría, no es posible determinar cuál es el papel que cumple la asimetría en la selección sexual (Swaddle, 1999; Schlaepfer $\&$ McNeil, 2000 para otros ejemplos). Según Swadle (2003), solamente seis estudios han investigado el efecto directo de la asimetría fluctuante en los procesos de selección sexual. De estos casos, tres mencionan que individuos con baja asimetría en los rasgos sexuales secundarios tienen una ventaja sobre sus compañeros asimétricos (Swaddle et al.; Møller \& Sorci, 1998; Morris \& Casey, 1998). Los tres estudios restantes indican que la asimetría fluctuante en otros caracteres sexualmente relacionados no tendría ninguna influencia perceptible sobre la selección de pareja o dominancia social (Swaddle \& Witter, 1995; Jablonski \& Matyjasiak, 1997; Tomkins \& Simmons, 1998).

Algunas conclusiones tempranas durante los años 90 sugerían que los niveles de AF podrían proporcionar una señal para la opción de la hembra en la selección sexual (Fitness) (Møller, 1990; Møller \& Hoglund 1991). Pero esta relación no siempre se encontró en estudios posteriores (Simmons et al. 1999), por lo que el papel exacto de AF en el proceso de selección sexual es un tema de mucho debate (Tomkins \& Simmons, 1998). Muchos estudios no han descubierto relaciones entre AF y varios componentes del fitness, lo que sugiere ser cuidadoso en el empleo de AF como un indicador total del fitness (Clarke 1995a; 1998; 2003; Leung \& Forbes, 2000).

En el caso de las señales (por ejemplo, los caracteres ornamentales) se ha demostrado una relación negativa entre la asimetría fluctuante y el tamaño del carácter en cuestión. Sólo los individuos capaces de soportar el estrés ambiental imperante pueden producir caracteres grandes y simétricos (Balmford et al., 1993; Evans, 1993; Møller \& Cuervo, 2003). Este patrón se predice porque si el tamaño del carácter es 
proporcional a los costos de la producción de este carácter, entonces sólo los individuos de alta calidad deberían ser capaces de producir caracteres sexuales grandes y simétricos (Tomkins \& Simmons, 1990; Møller \& Cuervo). Los caracteres ornamentales son más susceptibles al estrés ambiental que los caracteres morfológicos ordinarios, ya que estarían siendo afectados por una intensa selección sexual direccional que desestabilizaría el genoma (Møller \& Pomiankowski). Mientras, los caracteres ordinarios estarían sujetos a una selección estabilizadora. Por otro lado, debido a los elevados requerimientos fisiológicos y al desarrollo tan complejo que tienen los caracteres sexuales secundarios, serían más susceptibles de alterarse durante la morfogénesis que los caracteres morfológicos normales (Pomiankowski et al., 1991). Un ejemplo de esto se observó en la especie Chironomus plumosus L. (Díptera: Chironomidae) por McLachlan \& Cant (1995). Ellos detectaron una relación negativa entre el tamaño de los machos y el éxito reproductor. Además, los machos pequeños resultaron ser los más simétricos. Los autores explicaron estas relaciones negativas sobre la base de que los machos de $C$. plumosus forman enjambres que son visitados por las hembras para aparearse. En estos casos de emparejamiento en el aire existe una selección direccional sobre machos pequeños pues tienen mejor maniobrabilidad aérea y, por lo tanto, mayor éxito en la captura de hembras en vuelo. McLachlan \& Cant tomaron como medida de asimetría fluctuante la diferencia entre la longitud de las alas. Además de la asimetría absoluta utilizaron la asimetría relativa, calculada como la asimetría absoluta/longitud del ala, ya que esta medida sería adecuada para datos en los que muchos individuos no muestran diferencias medibles entre alas (Fig. 3).

Otro estudio en insectos, en donde se relaciona el tamaño de los rasgos morfológicos correlacionados con la selección sexual, es el de Blanckenhorn et al. (2003). Para detectar preferencia sexual de las hembras, estos autores evaluaron para diferentes rasgos morfológicos pareados la relación del tamaño del rasgo con la preferencia sexual en la especie Scathophaga stercoraria. Sus resultados iniciales indican que no existe selección sexual para los rasgos

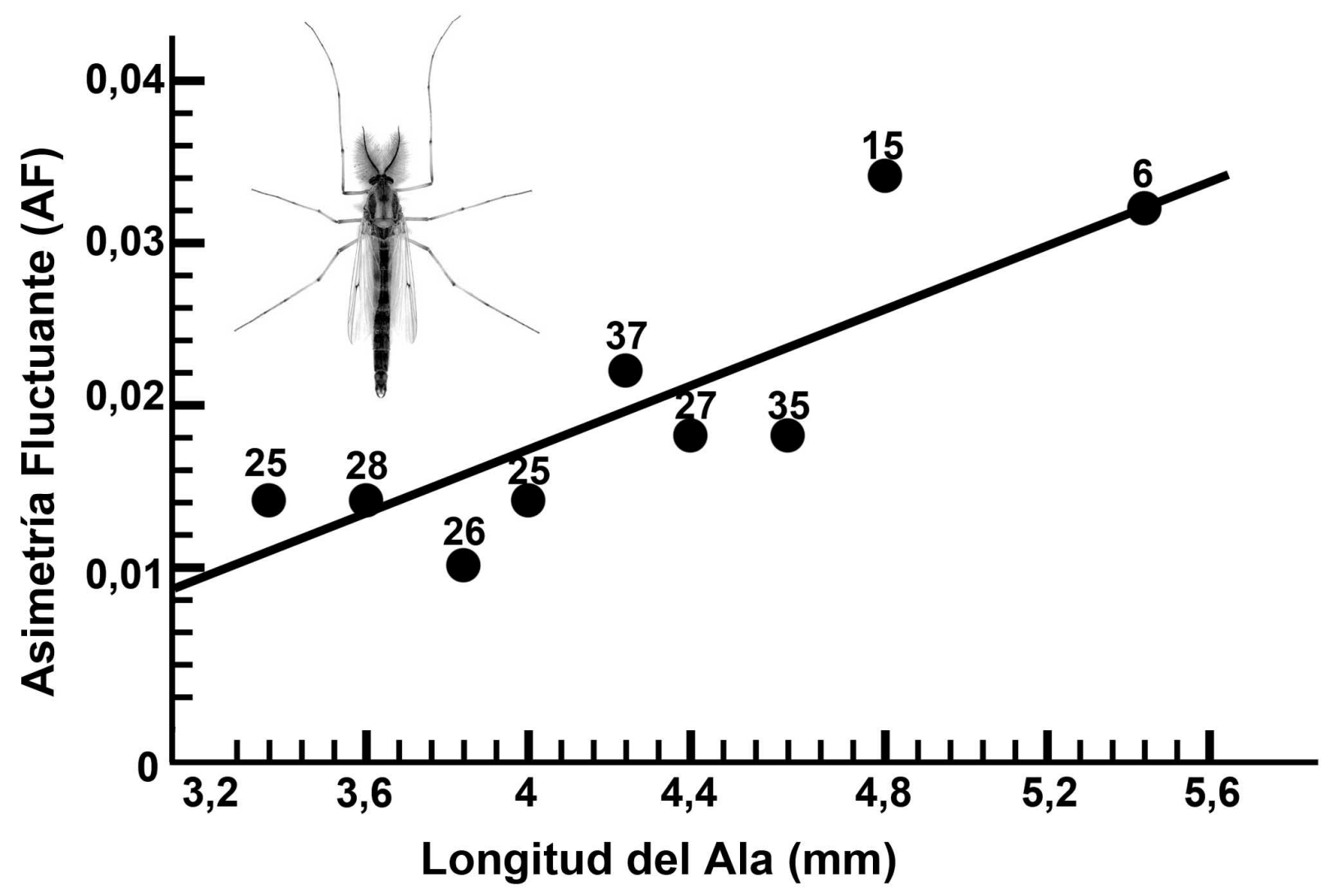

Fig. 3.- Relación entre asimetría fluctuante y longitud del ala en machos no emparejados de Chironomus plumosas. Los números representan el tamaño de muestra. Figura modificada de McLachan \& Cant (1995). 
pareados, entonces re-evaluaron correlacionando las muestras con rasgos fisiológicos de reservas energéticas (lípidos, glucosa y glicógeno). Estos son rasgos importantes para el éxito de apareamiento, además del tamaño de alas y patas por separado. Este ejemplo confirma la preferencia de hembras por machos grandes y con influencias positivas independientes para lípidos y glucosa. Blanckenhorn et al. concluyen que los rasgos pareados no afectan el éxito del apareamiento del macho y que la inclusión de rasgos fisiológicos serian útiles en selección sexual.

Genética de la Asimetría Fluctuante. La mayoría de los estudios empíricos para estimar la heredabilidad de AF son poco significativos (Leamy, 1999; Pelabon et al., 2004). Muy pocos presentan una heredabilidad significativa (Scheiner et al., 1991; Thornhill \& Sauer; 1992; Santos, 2002; Polak \& Starmer, 2005). Møller \& Thornhill (1997) para evaluar la heredabilidad global que existe para $\mathrm{AF}$, realizaron un meta-análisis para 34 estudios con heredabilidad de AF significativa (con un promedio de 0,19 sobre un número de caracteres y taxa). Sin embargo, las estimaciones de heredabilidad incluidos en estos 34 estudios, eran bastante deficientes en los diseños estadísticos y/o experimentales por lo que el meta-análisis ha inducido a malas interpretaciones (Markow \& Clarke, 1997; Palmer \& Strobeck, 1997). Los análisis posteriores muestran que la heredabilidad promedio de AF es probablemente menor (e.g. 0,08 Leamy, 1997; 0,043 Van Dongen \& Lens, 2000; 0,026 Fuller \& Houle, 2003). El valor de la heredabilidad promedio en estos estudios estaría puesto en duda, debido a que hay indicios de que la heredabilidad puede ser un rasgo específico (Woods et al.). Correspondería a un patrón que se puede esperar a partir del modelo de desarrollo de Klingenberg \& Nijhout (1999), el cual es actualmente aceptado debido a que la heredabilidad de FA es muy pequeña (Leamy \& Klingenberg). Por ejemplo, si aceptáramos que la asimetría fluctuante tiene un componente genético aditivo (Møller \& Thomhill), los individuos que escogen una pareja simétrica, que ha tenido un desarrollo estable, darán lugar generalmente a hijos simétricos. Por tanto, una preferencia por parejas simétricas es un mecanismo para obtener un beneficio indirecto en el éxito reproductivo (Møller \& Swaddle).

En los estudios genéticos sería relevante incorporar la búsqueda de loci asociados con la asimetría fluctuante (y la inestabilidad del desarrollo) bajo diferentes condiciones de estrés ambiental. En estos se podría dilucidar si la expresión genética varía por las condiciones ambientales y sólo en casos de alto estrés en donde los genes se verían activados para proteger el desarrollo (Rutherford \& Lindquist, 1998). Consecuentemente, esta línea de investigación podría ser importante al momento de evaluar los efectos directos del ambiente sobre los orígenes de asimetría fluctuante.
Asimetría Fluctuante + Morfometría Geométrica. En los últimos 12 años, en la búsqueda de nuevos métodos más precisos para cuantificar la variación de la asimetría en los individuos, la incorporación de la morfometría geométrica como herramienta ha permitido determinar y cuantificar cambios directamente desde la forma, reduciendo la tasa de error de medición presente frecuentemente en las mediciones con morfometría tradicional y permitiendo la estimación de variaciones más sutiles y exactas para el cálculo de asimetría (Klingenberg \& McIntyre; Auffray et al.; Zelditch et al., 2008). White \& Searle (2008), investigaron el uso de la morfometría geométrica a través de la asimetría fluctuante como proxy morfológico para cuantificar la diversidad genética de musarañas (Sorex araneus) en diferentes islas. Su estudio cuantifico la asimetría del lado izquierdo y derecho de la mandíbula de esta musaraña, en la que encontraron una pequeña relación entre la diversidad genética y la asimetría fluctuante de los individuos. La utilización de esta herramienta ha permitido cuantificar otros tipos de variaciones en el desarrollo, tales como cambios en la morfología en relación con la mutación en genes responsables del desarrollo. Fondon \& Garner (2007), hipotetizan que en cráneos de perros las simples asimetrías morfológicas son producidas por el incremento de mutaciones en el desarrollo (variaciones en factor de transcripción "Runx2" correlacionado con la sección facial del perro), lo que es confirmado a través de modelos de landmarks en 3D.

\section{DISCUSIÓN}

Las últimas tres décadas muestran un aumento progresivo de estudios en asimetría fluctuante (AF). La AF se usa como herramienta para evaluar la estabilidad durante el desarrollo, especialmente en el área de la biología evolutiva. A pesar de este largo período y muchos artículos publicados, el estado del conocimiento sobre la utilización de esta herramienta sólo permitiría, a la fecha, concluir que el modelo es heterogéneo y que se conoce muy poco sobre las causas subyacentes de esta heterogeneidad. Además, las propiedades estadísticas de AF siguen siendo ambiguas como causa de una insuficiente comprensión de los mecanismos subyacentes del tema (Van Dongen, 2006). Por tanto, es necesario un mayor esfuerzo en la comprensión de la heterogeneidad observada e intentar construir un protocolo estadístico más comprensible y unificador.

Uno de los temas más investigados actualmente, por la necesidad de comprender los mecanismos causales y sus efectos dentro de AF, son los aspectos moleculares, celulares y genéticos, en los cuales la evaluación de la validez en la heredabilidad de AF es un tema muy debatido (Leamy \& 
Klingenberg; Van Dongen, 2006). Møller \& Thornhill, en una revisión temprana de la literatura, señalan que AF muestra una pequeña pero significativa heredabilidad, en muchos taxa y sus atributos medibles. Sin embargo, muchos investigadores criticaron dichas aseveraciones señalado fallas en los análisis e interpretaciones de sus datos (Markow \& Clarke; Whitlock \& Fowler, 1997). Actualmente pareciera que existe una pequeña varianza genética aditiva debido a la asimetría fluctuante, pero no está claro como la heterogeneidad de la heredabilidad de AF es entre taxa y los caracteres (Van Dongen, 2000).

La incorporación de nuevas técnicas que determinan y sectorizan la variación de la forma está siendo actualmente un tema muy debatido (Lawing \& Polly, 2010; South \& Arnqvist, 2009), que a su vez sería -dentro de todas las metodologías morfométricas- a nuestro parecer, la más exacta e informativa en el momento de cuantificar una variación de asimetría. Trabajos han determinado relaciones entre la asimetría fluctuante de la forma y las variaciones en la diversidad genética, pero aún siguen siendo leves y casi nulos, debido a que no existe un patrón claro de método a seguir. Mezclar estas técnicas avanzadas del estudio de la morfometría, unificar metodologías junto a estudios moleculares y genéticos serían los pasos a los que los científicos deben apuntar, con el fin de obtener resultados de evidencia total dentro de los análisis.

Finalmente, esta revisión pretendió entregar una visión amplia del uso de AF para los diferentes ámbitos de la biología evolutiva; además, insistir en la necesidad de aumentar el esfuerzo de investigación y prestar mayor atención a la utilidad de AF como una medida de ED por su fácil, barata y rápida aplicación. Es necesario intensificar el esfuerzo de investigación y centrarse en los mecanismos subyacentes de ED, así como también en cómo estos procesos se asignan a los fenotipos observables. La idea es producir mejores modelos de estudio y lograr unificar enfoques para el análisis de los patrones en AF y ED.

AGRADECIMIENTOS: Los autores agradecen a Proyecto DIUC 210.113.079-1.0 de la Dirección de Investigación de la Universidad de Concepción, al Dr. Chris Klingenberg (University of Manchester) por la colaboración y comentarios en la sección Genética del artículo y a Luis Benítez de la Fuente por la gestión grafica del artículo.

BENITEZ, H. A. \& PARRA, L. E. Fluctuating asymmetry: A morpho-functional tool to measure developmental stability. Int. J. Morphol., 29(4):1459-1469, 2011.

SUMMARY: The ability of an organism to produce an ideal phenotype, despite the disturbances encountered during its development is the causal mechanism of the developmental stability (DS). This ability is used to evaluate a variety of stress types and the genotypic ability to correct them. The measurement tool mostly used to estimate the DS is the fluctuating asymmetry (FA), this being a small measure of random deviations that occur between the left and right sides of bilaterally symmetrical features. The study of FA management in its simplicity and speed in obtaining results has become the focus of interest in biology, and during the last years, and although there has been a progressive increase in published articles using FA to evaluate disturbances in the DS, many questions remain as to what constitutes the underlying causes that generate FA. This review compiles information on the progress in studies of FA use, its methodology, mechanisms, benefits and controversies in time, and proposes that it is strictly necessary to generate a better understanding of this tool, and as a result the use of more precise study models that justify a unified approach to the analysis of patterns in FA and DS.

KEY WORDS: Developmental instability; Sexual Selection; Genotype; Phenotype.

\section{REFERENCIAS BIBLIOGRÁFICAS}

Auffray, J.; Debat, V. \& Alibert, P. Shape asymmetry and developmental stability. In: Chaplain, M. A.; Singh, G. D. \& McLachlan, J. C. On growth and form: spatio-temporal pattern formation in biology. Chichester, John Wiley \& Son, 1999. pp. 309-24.

Balmford, A.; Jones, I. L. \& Thomas, A. L. On avian asymmetry evidence of natural selection for symmetrical tails and wings in birds. P. Roy. Soc. Lond. B Bio., 252:245-51, 1993.

Benítez, H.; Briones, R. \& Jerez, V. Asimetría Fluctuante en dos poblaciones de Ceroglossus chilensis (Coleoptera Carabidae) en el agroecosistema Pinus radiata, Región del BioBío. Gayana, 72(2):131-9, 2008.

Bjorksten, T.; David, P.; Pomiankowski, A. \& Fowler, K. Fluctuating asymmetry of sexual and nonsexual traits in stalkeyed ies: A poor indicator of developmental stress and genetic quality. J. Evolution Biol., 13:89-97, 2000.

Blanckenhorn, U.; Karaushaar, U. \& Reim, C. Sexual selection on morphological and physiological traits and fluctuating asymmetry in the yellow dung fly. J. Evolution Biol., 16:90313, 2003. 
Clarke, G. M. Relationships between developmental stability and fitness: application for conservation biology. Conserv. Biol., 9:18-24, 1995a.

Clarke, G. M. Relationships between fluctuating asymmetry and fitness: how good is the evidence? P. Conserv. Biol., 2:146-9, 1995b.

Clarke, G. M. Developmental stability and fitness: the evidence is not quite so clear. Am. Nat., 152:2-766, 1998.

Clarke, G. M. Developmental stability - fitness relationships in animals: some theoretical considerations. In: Polak, M. Developmental Instability: Causes and Consequences, Oxford, Oxford University Press, 2003. pp. 187-95.

Evans, M. R. Fluctuating asymmetry and long tails: the mechanical effects of asymmetry may act to enforce honest advertisement. P. Roy Soc. Lond. B Bio., 253:205-9, 1993.

Fields, S.; Spiers, M.; Herschkovitz, I. \& Livshits, G. Reliability of reliability coefficients in the estimation of asymmetry. Am. J. Phys. Anthropol., 96:83-7, 1995.

Fondon, J. W. \& Garner, H. R. Detection of lengthdependent effects of tandem repeat alleles by 3-D geometric decomposition of craniofacial variation. Dev. Genes Evol., 217:79-85, 2007.

Fuller, R. C. \& Houle, D. Inheritance of developmental instability. In: Polak, M. Developmental Instability: Causes and Consequences. Oxford, Oxford University Press, 2003. pp.157-86.

Fumi, M. Distinguishing between Leptidea sinapis and L. reali (Lepidoptera: Pieridae) using a morphometric approach: impact of measurement error on the discriminative characters. Zootaxa, 1819:40-54, 2008.

Graham, J. H.; Fletcher, D.; Tigue, J. \& McDonald, M. Growth and developmental stability of Drosophila melanogaster in low frequency magnetic fields. Bioelectromagnetics, 21:465-72, 2000.

Greene, D. L. Fluctuating dental asymmetry and measurement error. Am. J. Phys Anthropol., 65:283-9, 1984.

Hoffmann, A. A. \& Woods, R. E. Associating environmental stress with developmental stability: problems and patterns. In: Polak, M. Developmental Instability: Causes and Consequences, Oxford, Oxford University Press, 2003. pp. 387-401.

Jablonski, P. G. \& Matyjasiak, P. Chaffinch (Fringilla coelebs) epaulette display depends on the degree of exposure but not symmetry of intruder's epaulettes. Behaviour, 134:1115-21, 1997.

Klingenberg, C. P. A developmental perspective on developmental instability: theory, models, and mechanisms. In: Polak, M.
Developmental Instability: Causes and Consequences. Oxford, Oxford University Press, 2003. pp. 13-34.

Klingenberg, C. P. \& McIntyre, G. S. Geometric morphometrics of developmental instability: Analyzing patterns of fluctuating asymmetry with Procrustes methods. Evolution, 52:1363-75, 1998.

Klingenberg, C. P. \& Nijhout, H. F. Genetics of fluctuating asymmetry: a developmental model of developmental instability. Evolution, 53:358-75, 1999.

Klingenberg, C. P.; Badyaev, A. V.; Sowry, S. M. \& Beckwith, N. J. Inferring developmental modularity from morphological integration: Analysis of individual variation and asymmetry in bumblebee wings. Am. Nat., 157:11-23, 2001.

Lawing, A. M. \& Polly, P. D. Geometric morphometrics: recent applications to the study of evolution and development. J. Zool., $280: 1-7,2010$

Leamy, L. J. Is developmental stability heritable? J. Evolution Biol., 10:21-9, 1997.

Leamy, L. J. Heritability of directional and fluctuating asymmetry for mandibular characters in random-bred mice. J. Evolution Biol., 12:146-55, 1999.

Leamy, L. J. \& Klingenberg, C. P. The genetics and evolution of fluctuating asymmetry. Annu. Rev. Ecol. Syst., 36:1-21, 2005.

Leung, B.; Forbes, M. R. \& Houle, D. Fluctuating asymmetry as a bioindicator of stress: comparing efficacy of analyses involving multiple traits. Am. Nat., 155:101-15, 2000.

Mardia, K. V.; Bookstein, F. L. \& Moretón, I. J. Statistical assessment of bilateral symmetry of shapes. Biometrika, 87:285-300, 2000.

Markow, T. A. \& Clarke, G. M. Meta-analysis of the heritability of developmental stability: a giant step backward. J. Evolution Biol., 10:31-7, 1997.

McLachlan, A. \& Cant, M. Small males are more symmetrical: mating success in the midge Chironomus plumosus L. (Diptera: Chironomidae). Anim. Behav., 50:841-6, 1995.

Merilä, J. \& Björklund, M. Fluctuating asymmetry and measurement error. Syst. Biol., 44:97-101, 1995.

Møller, A. P. Fluctuating asymmetry in male sexual ornaments may reliably reveal male quality. Anim. Behav., 40:1185-7, 1990.

Møller, A. P. Sexual selection in the barn swallow (Hirundo rustica) IV. Patterns of fluctuating asymmetry and selection against asymmetry. Evolution, 48:658-70, 1994.

Møller, A. P. \& Hoglund, J. Patterns of fluctuating asymmetry in 
avian feather ornaments: Implications for models of sexual selection. P. Roy Soc. Lond. B Bio., 254(1312):1-5, 1991.

Møller, A. P. \& Pomiankowski, A. Fluctuating asymmetry and sexual selection. Genetica, 89:267-79, 1993.

Møller, A. P. \& Sorci, G. Insect preference for symmetrical artificial flowers. Oecologia, 114:37-42, 1998.

Møller, A. P. \& Swaddle, J. P. Asymmetry, Developmental Stability and Evolution. Oxford, Oxford Univ. Press, 1997.

Møller, A. P. \& Thornhill, R. A meta-analysis of the heritability of developmental stability. J. Evolution Biol., 10:1-16, 1997.

Møller, A. P. \& Cuervo, J. J. Asymmetry, size, and sexual selection: factors affecting heterogeneity in relationships between asymmetry and sexual selection. In: Polak, M. Developmental Instability: Causes and Consequences. Oxford, Oxford University Press, 2003. pp.262-275.

Møller, A. P. \& Thornhill, R. Bilateral symmetry and sexual selection: A meta-analysis. Am. Nat., 151:174-92, 1998.

Morris, M. R. \& Casey, K. Female swordtail fish prefer symmetrical sexual signal. Anim. Behav., 55:33-9, 1998.

Muñoz-Muñoz, F. \& Perpiñan, D. Measurement error in morphometric studies: comparison between manual and computerized methods. Ann. Zool. Fenn., 47:46-56, 2010.

Nijhout, H. F. \& Davidowitz, G. Developmental perspectives on phenotypic instability, canalization, and fluctuating asymmetry. In: Polak, M. Developmental Instability: Causes and Consequences. Oxford, Oxford University Press, 2003. pp. 313.

Palmer, A. R. Fluctuating asymmetry analyses: a primer. In: Markow, T. A Developmental instability: its origins and implications. The Netherlands, Kluwer, Dordrecht, 1994. pp. 335-64.

Palmer, A. R. Waltzing with asymmetry: is fluctuating asymmetry a powerful new tool for biologists or just an alluring new dance step? BioScience, 46:518-32, 1996.

Palmer, A. R. \& Strobeck, C. Fluctuating asymmetry analysis revisited. In: Polak, M. Developmental Instability: Causes and Consequences. Oxford, Oxford University Press, 2003. pp. 279-319.

Palmer, A. R, \& Strobeck, C. Fluctuating asymmetry: measurement, analysis, patterns. Annu. Rev. Ecol Syst., 17:391421, 1986.

Palmer, A. R. \& Strobeck, C. Fluctuating asymmetry and developmental stability: heritability of observable variation vs. heritability of inferred cause. J. Evolution Biol., 10:39-49, 1997.
Pankakoski, E.; Koivisto, I. \& Hyvarinen, H. Reduced developmental stability as an indicator of heavy metal pollution in the common shrew, Sorex araneus. Acta Zool. Fenn., 191:137-44, 1992.

Parsons, P. A. Fluctuating asymmetry: A biological monitor of environmental and genomic stress. Heredity, 68:361-4, 1992.

Pelabon, C.; Hansen, T. F.; Carlson, M. L. \& Armbruster, W. S. Variational and genetic properties of developmental stability in Dalechampia scandens. Evolution, 58:504-14, 2004.

Pither, J. \& Taylor, P. D. Directional and fluctuating asymmetry in the black-winged damselfly Calopteryx maculate (Beauvois) (Odonata: Calopterygidae). Can J. Zool., 78:1740-8, 2000.

Pomiankowski, A. Iwasa, Y. \& Nee, S. The evolution of costly mate preferences. I. Fisher and biased mutation. Evolution, 45:1422-30, 1991.

Polak, M. Developmental Instability. Causes and Consequences. New York, Oxford Univ. Press, 2003.

Polak, M. \& Starmer, W. T. The quantitative genetics of fluctuating asymmetry. Evolution, 55:498-511, 2001.

Polak, M. \& Starmer, W. T. Environmental origins of sexually selected variation and a critique of the fluctuating asymmetrysexual selection hypothesis. Evolution, 59:577-85, 2005.

Rutherford, S. L. \& Lindquist, S. Hsp90 as a capacitor for morphological evolution. Nature, 396:336-42, 1998.

Santos, M. Genetics of wing size asymmetry in Drosophila buzzatii. J. Evolution Biol., 15:720-34, 2002.

Scheiner, S. M.; Caplan, R. L. \& Lyman, R. F. The genetics of phenotypic plasticity. III. Genetic correlations and fluctuating asymmetries. J. Evolution Biol., 4:51-68, 1991.

Schlaepfer, M. A. \& McNeil, J. N. Are virgin male lepidopterans more successful in mate acquisition than previously mated individuals? A study of the European corn borer, Ostrinia nubilalis (Lepidoptera: Pyralidae). Can. J. Zool., 78:2045-50, 2000.

Simmons, L. W.; Tomkins, J. L.; Kotiaho, J. S. \& Hunt, J. Fluctuating paradigm. P. Roy Soc. Lond. B Bio., 266:593-5, 1999.

South, S. H. \& Arnqvis, G. Morphological variation of an ornament expressed in both sexes of the mosquito Sabethes cyaneus. Evol. Ecol Res., 11:1-21, 2009.

Swaddle, J. P.; Witter, M. \& Cuthill, I. C. The analysis of fluctuating asymmetry. Anim. Behav., 48:986-9, 1994.

Swaddle, J. P. \& Witter, M. S. Chest plumage, dominance and 
fluctuating asymmetry in female stariings. P. Roy Soc. Lond. B Bio., 260:219-23, 1995.

Swaddle, J. P. Is fluctuating asymmetry a visual signal? In: Espmark, Y.; Amundsen, T. \& Rosenqvist, G. Animal Signáis. Trondheim, Tapir Academic Press, 1999. pp. 155-75.

Swaddle, J. P. Fluctuating asymmetry, animal behavior, and evolution. Adv. Stud. Behav., 32:169-20, 2003.

Thornhill, R. \& Sauer, P. Genetic sire effects on the fighting ability of sons and daughters and mating success of sons in a scorpionfly. Anim. Behav., 43:255-64, 1992.

Thornhill, R.; Møller, A. P. \& Gangestad, S. W. The biological significance of fluctuating asymmetry and sexual selection: a reply to Palmer. Am. Nat., 154:234-41, 1999.

Tomkins, J. L. \& Simmons, L. W. Female choice and manipulations of forceps size and symmetry in the earwig Forficula auricularia L. Anim Behav., 56:347-56, 1998.

Tomkins, J. L. \& Simmons, L. W. Fluctuating asymmetry and sexual selection: paradigm shifts, publication bias, and observer expectation. In: Polak, M. Developmental Instability: Causes and Consequences, Oxford University Press, Oxford, 2003. pp. 231-61.

Van Dongen, S. How repeatable is the estimation of developmental stability by fluctuating asymmetry? P. Roy Soc. Lond. B Bio., 265:1423-27, 1998.

Van Dongen, S. Fluctuating asymmetry and developmental instability in evolutionary biology: past, present and future. $J$. Evolution Biol., 19:1727-43, 2006.

Van Dongen, S. \& Lens, L. The evolutionary potential of developmental instability. J. Evolution Biol., 13:326-35, 2000.

Van Valen, L. A study of fluctuating asymmetry. Evolution, 16:12542, 1962.

Waddington, C. H. Canalization of development and the inheritance of acquired characters. Nature, 150:563-5, 1942.

White, T. A. \& Searle, J. B. Mandible asymmetry and genetic diversity in island populations of the common shrew, Sorex araneus. J. Evolution Biol., 21:636-41, 2008.

Whitlock, M. C, \& Fowler, K. The instability of studies of instability. J. Evolution Biol., 10:63-7, 1997.

Windig, J. \& Nylin, S. How to compare fluctuating asymmetry of different traits. J. Evolution Biol., 13:29-37, 2000.

Woods, R. E.; Sgro, C. M.; Hercus, M. J. \& Hoffmann, A. A. The association between fluctuating asymmetry, trait variability, trait heritability, and stress: A multiply replicated experiment on combined stresses in Drosophila melanogaster. Evolution, 53:493-505, 1999.

Zelditch, M. L.; Wood, A. R. \& Swiderski, D. L. Building developmental integration into functional systems: functioninduced integration of mandibular shape. Evol. Biol., 36:7187, 2008.

\author{
Dirección para correspondencia: \\ Luis E. Parra \\ Departamento de Zoología \\ Facultad de Ciencias Naturales y Oceanográficas \\ Universidad de Concepción \\ Casilla 160-C \\ Concepción \\ CHILE
}

E-mail: luparra@udec.cl

Recibido : 02-08-2011

Aceptado: 06-09-2011 